\title{
Laser-wakefield acceleration of electron beams in a low density plasma channel
}

\author{
T. P. A. Ibbotson, ${ }^{1}$ N. Bourgeois, ${ }^{1}$ T. P. Rowlands-Rees,${ }^{1}$ L. S. Caballero, ${ }^{1}$ S. I. Bajlekov, ${ }^{1}$ P. A. Walker, ${ }^{1}$ \\ S. Kneip, ${ }^{2}$ S. P. D. Mangles, ${ }^{2}$ S. R. Nagel, ${ }^{2}$ C. A. J. Palmer, ${ }^{2}$ N. Delerue, ${ }^{3}$ G. Doucas, ${ }^{3}$ D. Urner,${ }^{3}$ O. Chekhlov, ${ }^{4}$ \\ R. J. Clarke, ${ }^{4}$ E. Divall, ${ }^{4}$ K. Ertel, ${ }^{4}$ P. S. Foster,${ }^{4}$ S. J. Hawkes, ${ }^{4}$ C. J. Hooker ${ }^{4}$ B. Parry, ${ }^{4}$ P. P. Rajeev, ${ }^{4}$ \\ M. J. V. Streeter, ${ }^{4}$ and S. M. Hooker ${ }^{1}$ \\ ${ }^{1}$ Clarendon Laboratory, Parks Road, Oxford, OX1 3PU, United Kingdom \\ ${ }^{2}$ The Blackett Laboratory, Imperial College London, SW7 2BZ, United Kingdom \\ ${ }^{3}$ John Adams Institute for Accelerator Science, University of Oxford, Oxford, United Kingdom \\ ${ }^{4}$ Central Laser Facility, Rutherford Appleton Laboratory, Oxon, OX11 OQX, United Kingdom
}

(Received 10 August 2009; published 17 March 2010)

\begin{abstract}
The generation of quasimonoenergetic electron beams, with energies greater than $500 \mathrm{MeV}$, in a laserplasma accelerator driven by $2.5 \mathrm{~J}, 80 \mathrm{fs}$ laser pulses guided in a low density plasma channel, is investigated. The laser energy required to achieve electron injection is found to depend strongly on the quality of the input laser focal spot. Simulations show that, although the matched spot size of the plasma channel is greater than the self-focused spot size, the channel assists relativistic self-focusing and enables electron injection to occur at lower plasma densities and laser powers than would be possible without a waveguide.
\end{abstract}

DOI: 10.1103/PhysRevSTAB.13.031301

PACS numbers: $52.38 . \mathrm{Kd}, 41.75 . \mathrm{Jv}, 52.38 . \mathrm{Hb}$

In a laser-plasma accelerator, an intense laser beam is used to drive a relativistic plasma wave, within which are formed strong longitudinal electric fields able to accelerate beams of charged particles [1]. This approach has been used to generate quasimonoenergetic beams of electrons from plasma accelerators driven in plasma channels [2-4] or gas jets [5-7] to energies as high as $1 \mathrm{GeV}[3,4]$.

Most experiments to date have operated in the nonlinear regime, characterized by a normalized vector potential $a_{0}>1$, where $a_{0}=e A_{0} / m_{e} c$ and $A_{0}$ is the peak vector potential of the laser field. For sufficiently high peak laser powers $P_{0}$, and for $a_{0}>2$, the bubble (or blowout) regime $[8,9]$ is reached in which essentially all the plasma electrons are expelled from an approximately spherical region immediately behind the laser pulse. Electrons from the plasma can be trapped within this cavity and accelerated until either they move into a decelerating phase of the wakefield, which occurs after acceleration over a distance $L_{d}=(4 / 3)\left(\omega_{0}^{2} c / \omega_{p}^{3}\right) \sqrt{a_{0}}$, or until the pump laser depletes, which occurs after propagation over $L_{p d}=\left(\omega_{0} / \omega_{p}\right)^{2} c \tau$ [8]. Here $\omega_{0}=2 \pi c / \lambda_{0}$, where $\lambda_{0}$ is the wavelength of the driving laser; $\tau$ is the full-width at half-maximum (FWHM) duration of the laser pulse; and $\omega_{p}=$ $\left(n_{e} e^{2} / m_{e} \epsilon_{0}\right)^{1 / 2}$, where $n_{e}$ is the electron density.

In the bubble regime relativistic self-focusing plays an important role in keeping the spot size of the laser of the order of the plasma wavelength. The condition for self-focusing is usually taken to be $P_{0}>P_{c}=$ $17.4\left(\omega_{0} / \omega_{p}\right)^{2} \mathrm{GW}$. However, transverse density gradients at the front of the laser pulse almost cancel self-focusing so that the front of the pulse diffracts essentially freely. Lu et al. have shown [8] that the laser pulse and bubble will propagate stably if: (i) the laser spot size $w_{0} \approx w_{s f} \equiv$
$2 k_{p}^{-1} \sqrt{a_{0}}$, where $k_{p}=\omega_{p} / c$; (ii) the rate of erosion of the leading edge of the pulse by diffraction is slower than that caused by transfer of laser energy to the plasma wave. In the absence of any external waveguide, the condition for self-guiding (ii) becomes $P_{0} \geqq P_{c}^{\text {diff }}$, where $P_{c}^{\text {diff }}=$ $(1 / 8)\left(\omega_{0} / \omega_{p}\right)^{6 / 5} P_{c}$. A waveguide with a matched spot size $w_{m}=w_{s f}$ relaxes condition (ii) to $P_{0} \gtrsim P_{c}$ as diffraction of the leading edge is eliminated.

In this paper we report experiments in which electron beams are generated in plasma channels with matched spot sizes $w_{m}>w_{s f}$. We show through simulations that even in this case, diffraction is suppressed sufficiently for a laser pulse with $P_{0} \lesssim P_{c}^{\text {diff }}$ (but $P_{0} \gtrsim P_{c}$ ) to evolve to one which propagates stably with a spot size close to $w_{s f}$, and that this enables electron injection at lower laser powers and plasma densities than possible without a waveguide. In our experiments we observe the transition between relativistically guided and channel-assisted guiding in the behavior of the generated electron beams. We also show that improving the transverse profile of the driving laser pulse can reduce significantly the laser energy required to achieve electron injection.

In this work the plasma channel was formed by a hydrogen-filled capillary discharge waveguide, as described in detail elsewhere [10,11]. The capillary was $33 \mathrm{~mm}$ long and $200 \mu \mathrm{m}$ in diameter; slots of diameter $650 \mu \mathrm{m}$ were machined $4 \mathrm{~mm}$ from the ends to allow $\mathrm{H}_{2}$ gas to flow into the capillary. A discharge current was driven through the capillary by connecting a $1.7 \mathrm{nF}$ capacitor, charged to $25 \mathrm{kV}$, across electrodes located at each end; the resulting current pulse peaked at $\sim 500 \mathrm{~A}$ and was of duration $\sim 200$ ns. Previous work [12] has shown that the plasma channel evolves slowly near the peak of the 


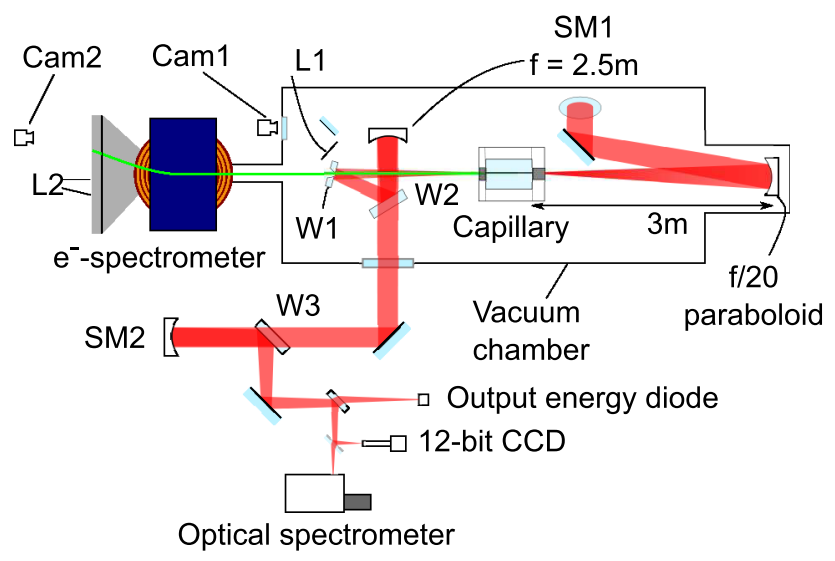

FIG. 1. (Color) Schematic diagram of the experimental layout.

current pulse, with an axial electron density $n_{e}(0)$ which depends only on the initial $\mathrm{H}_{2}$ pressure $P$. In the experiments reported here this ranged from $n_{e}(0)=1.2 \times$ $10^{18}-4.4 \times 10^{18} \mathrm{~cm}^{-3}$ for $P=50-200 \mathrm{mbar}$, and for these pressures $n_{e}(0)$ varies linearly with $P$.

The experiments were performed with the Astra-Gemini laser, which for this work delivered pulses with full-width at half-maximum (FWHM) duration of 80 fs and a central wavelength of $780 \mathrm{~nm}$. A simplified diagram of the experimental layout is shown in Fig. 1. An $f / 20$ off-axis paraboloid, with a focal length of $3 \mathrm{~m}$, focused the beam to a spot size of $22 \mu \mathrm{m}$ (FWHM) at the entrance to the capillary. For the range of initial $\mathrm{H}_{2}$ pressures investigated, the FWHM of the lowest-order mode of the plasma channel ranged from 32-42 $\mu \mathrm{m}$ [12]. To better match the input spot, and to improve the focal spot quality, a soft aperture was placed in the amplifier chain; this increased the size of the focal spot to $35 \mu \mathrm{m}$ (FWHM), and removed structure in the transverse wings of the focal spot, but limited the maximum laser energy on target to $3 \mathrm{~J}$.

Laser radiation transmitted by the waveguide was reflected from optically flat wedges W1 and W2, and collimated by reflection at normal incidence from a spherical mirror (SM1). The intensity of this parallel beam was further reduced by a reflection from another wedge and transmitted out of the vacuum chamber. This beam was refocused by a second combination of a wedge (W3) and spherical mirror (SM2), further reduced in intensity by reflections from a wedge and a dielectric beam splitter, and imaged onto a 12-bit CCD camera by a $\times 5$ microscope objective. The beams transmitted by the wedge and beam splitter were imaged onto a photodiode and optical spectrometer to give the energy and spectrum of the laser radiation.

Electron beams generated within the capillary discharge passed through a $5 \mathrm{~mm}$ diameter hole in $\mathrm{W} 1$, were dispersed by an electron spectrometer, and detected by a phosphor (Lanex) screen (L2) imaged by a 12-bit CCD camera. The electron spectrometer used an electromagnet to disperse the electron beams, which could produce mag- netic fields of up to $1.2 \mathrm{~T}$ and had an effective field radius $r_{\text {eff }}=167 \mathrm{~mm}$. The wedge $\mathrm{W} 1$ could be replaced by a Lanex screen (L1), allowing the electron beam profile and electron energy spectrum to be recorded simultaneously. The charge of the electron bunches reaching the electron spectrometer was determined by cross calibrating the fluorescence signal produced by L 2 with the signal produced by a calibrated image plate (Fuji BAS MS).

The delay $t_{d}$ between the onset of the discharge current and the arrival of the laser pulse was measured by a fast photodiode and Rogowski coil.

We first present results obtained with the aperture in position and with a mean on-target pulse energy of $(2.53 \pm$ $0.15) \mathrm{J}$ and laser pulse duration of $(80 \pm 5) \mathrm{fs}$, corresponding to a peak normalized vector potential $a_{0} \approx 0.9$ at the entrance of the capillary.

Figure 2 shows the total electron charge, as measured on $\mathrm{L} 1$, against $t_{d}$. As L1 was located before the electron spectrometer, electrons at all energies contribute to this value. The inset shows the delay at which electron beams were first observed and at which the highest bunch charge was recorded. It is seen that the electron beam generation is quite different for $P \gtrsim 100$ mbar than at lower pressures: for $P \leqq 100$ mbar electron beams are not recorded until $t_{d} \gtrsim 150 \mathrm{~ns}$, while for higher pressures they are observed shortly after the breakdown phase of the discharge. It is noticeable that for $P=80$ and 110 mbar the total charge of the electron beams shows a well-defined optimum delay; whereas for $P=200 \mathrm{mbar}$, beams of similar charge are generated for a wide range of delays.

Figure 3 shows, for several values of $P$, measured electron energy spectra at delays $t_{d}$ corresponding to optimum beam generation. It may be seen that the beam energy is maximized, and the energy spread minimized, for

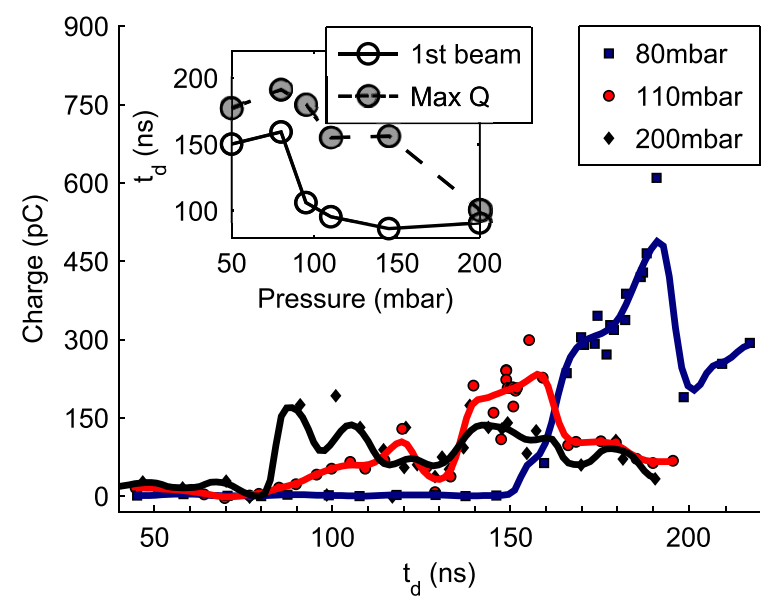

FIG. 2. (Color) Total electron beam charge $Q$ measured on L1 as a function of delay $t_{d}$ for $P=80,110,200$ mbar. The solid lines are a moving average using a Gaussian function, defined by $e^{-t_{d}^{2} / \tau^{2}}$, where $\tau=5 \mathrm{~ns}$. Inset: Delay $t_{d}$ at which electron beams were first observed, and delay at which the highest bunch charge $Q$ was observed, as a function of $P$. 


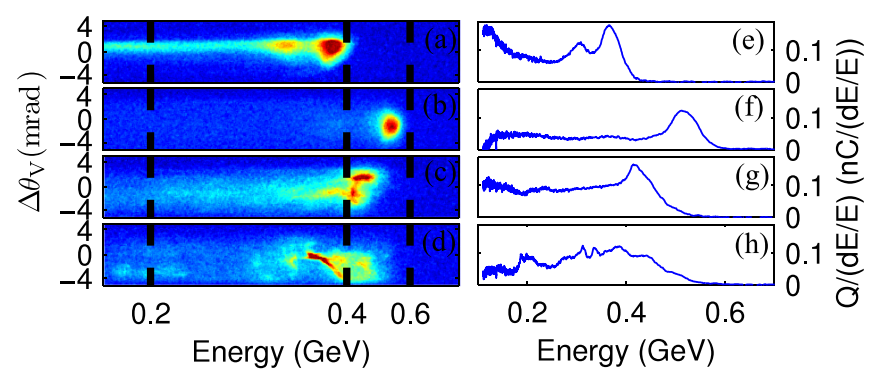

FIG. 3. (Color) Raw electron energy spectra (a)-(d) showing the fluorescence from L2, and spectra in units of charge per relative energy spread (e)-(h) for: (a),(e) $P=50$ mbar, $t_{d}=147 \mathrm{~ns}$; (b),(f) $P=80$ mbar, $t_{d}=189 \mathrm{~ns}$; (c),(g) $P=110$ mbar, $t_{d}=$ $181 \mathrm{~ns}$; (d),(h) $P=200$ mbar, $t_{d}=150 \mathrm{~ns}$.

$P \approx 80$ mbar. The beam shown in Fig. 3(b) has an energy of $520 \mathrm{MeV}$ and a charge of $70 \mathrm{pC}$.

At lower pressures the electron beam divergence was smaller, however the pointing fluctuations were larger. For example, for the beams shown in Fig. 2 at 80 mbar, the mean beam divergence ( $1 / e$ full width) in the horizontal $\left(\Delta \theta_{\mathrm{H}}\right)$ and vertical $\left(\Delta \theta_{\mathrm{V}}\right)$ directions was $(3.9 \pm 0.8) \mathrm{mrad}$ and $(5.4 \pm 1.3) \mathrm{mrad}$, respectively, where the errors are the standard deviation; the rms variation in beam pointing was 4.6 and $2.4 \mathrm{mrad}$ in the horizontal $\left(\sigma_{\mathrm{H}}\right)$ and vertical $\left(\sigma_{\mathrm{V}}\right)$ directions. In comparison, at $200 \mathrm{mbar}, \Delta \theta_{\mathrm{H}}=(13.9 \pm$ 3.7) $\mathrm{mrad}$ and $\Delta \theta_{\mathrm{V}}=(16.0 \pm 3.7) \mathrm{mrad}$ with $\sigma_{\mathrm{H}}=$ $1 \mathrm{mrad}$ and $\sigma_{\mathrm{V}}=1.5 \mathrm{mrad}$.

Figure 4 compares the total electron bunch charge measured by L1 as a function of the laser pulse energy on target, with and without the aperture in position. It is seen that introducing the aperture reduced the threshold energy for generating electron beams from $3.5 \mathrm{~J}$ to less than $2 \mathrm{~J}$. In both cases $E_{\max }>500 \mathrm{MeV}$.

The results obtained may be interpreted with the model of Lu et al. [8]. For $P=80 \mathrm{mbar}\left(n_{e}=1.8 \times 10^{18} \mathrm{~cm}^{-3}\right)$, we find $P_{0} \approx 1.7 P_{c}$ and $P_{c}^{\text {diff }}=7.7 P_{c}$, and hence the condition for self-guiding is not satisfied. In contrast, for

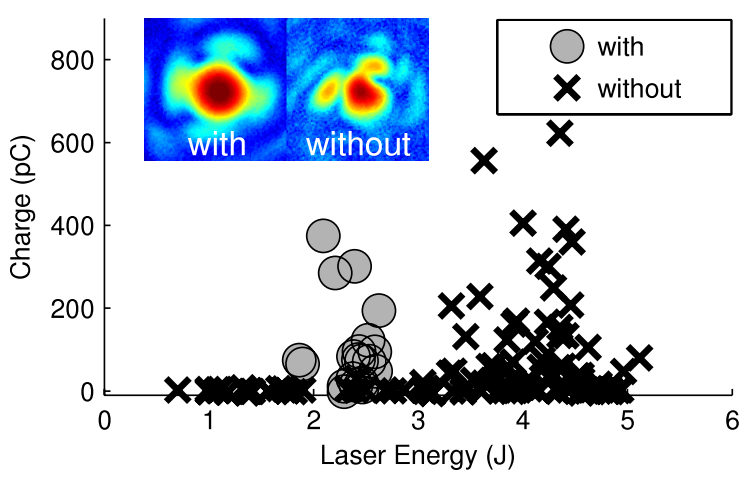

FIG. 4. (Color) Total beam charge $Q$ measured on L1 as a function of input laser energy with and without the aperture in position, for $P=95$ mbar. Inset: Images of the focal spot with and without the aperture, on a logarithmic scale to emphasize features in the wings of the pulse.
$P=200$ mbar $\left(n_{e}=4.4 \times 10^{18} \mathrm{~cm}^{-3}\right)$ we have $P_{0} \approx$ $4.2 P_{c}$ and $P_{c}^{\text {diff }}=4.5 P_{c}$, which is close to satisfying the self-guiding condition. Thus, it would be expected that for $P \gtrsim 200$ mbar relativistic self-focusing would play a bigger role in the laser propagation than the plasma channel, and hence the propagation of the laser pulse would be similar to that in a gas jet or cell at the same density. However, at lower densities the plasma channel should play a more significant role in the propagation of the driving laser pulses, and in the development of the wakefield. Given that guiding is required to reach the conditions for electron injection, as discussed below, this explains the observation that for $P \approx 200$ mbar electron beams were generated over a wide range of delays $t_{d}$-behavior more characteristic of a gas cell-whereas at lower pressures injection was much more sensitive to the delay $t_{d}$, consistent with an evolving plasma channel. The sensitivity to $t_{d}$ may be caused by evolution of the longitudinal and transverse density profiles of the plasma channel; it has also been shown that ionization might assist electron injection at delays $t_{d}$ for which the plasma is partially ionized [13].

The role of the plasma channel at low densities is confirmed by simulations with the 3D axisymmetric particlein-cell (PIC) code WAKE [14], as shown Fig. 5. We see that

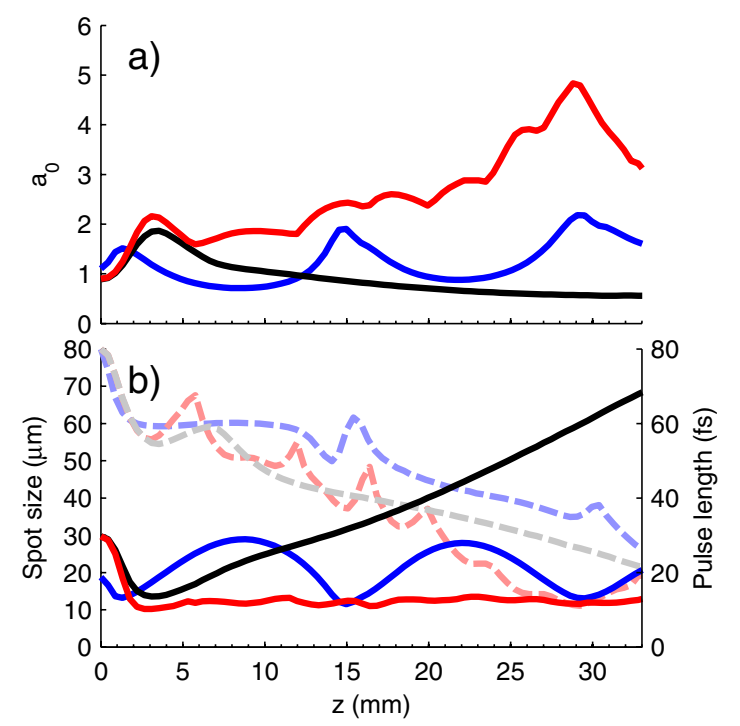

FIG. 5. (Color) Calculated values, as a function of propagation distance $z$, of: (a) peak $a_{0}$, (b) mean spot radius ( $\left.\frac{1}{e^{2}}\right)$ (solid) and FWHM duration (dashed) of laser pulses propagating through a gas cell (black) and a plasma channel (red/blue). For these calculations the channel was parabolic, with $n_{e}(0)=$ $1.8 \times 10^{18} \mathrm{~cm}^{-3}$ and lowest-order mode of $41 \mu \mathrm{m}$ FWHM, corresponding to $P=80 \mathrm{mbar}$; the gas in the cell was fully ionized, with an electron density of $n_{e}(0)$. The temporal and transverse spatial profiles of the input laser pulses were Gaussian, with initial FWHM duration of 80 fs and FWHM focal spots of $35 \mu \mathrm{m}$ (black/red) and $22 \mu \mathrm{m}$ (blue). The input laser energies were adjusted to give the measured input fluences when the total on-target laser energy was $2.5 \mathrm{~J}$, i.e., 2 and $1.2 \mathrm{~J}$ with and without the aperture, respectively. 
for $P=80$ mbar the propagation of the laser pulse is similar for a plasma channel and gas cell up to $z \approx$ $5 \mathrm{~mm}$; at this point, for both cases, $w_{0} \approx w_{s f}=12 \mu \mathrm{m}$ and $a_{0} \approx a_{s f}=2.4$, where $a_{s f}$ is the normalized vector potential for a pulse of spot size $w_{s f}$. Beyond this the channel assists relativistic self-focusing in maintaining an almost constant mean spot size $w_{0} \approx w_{s f}$, whereas in a gas cell the pulse diffracts rapidly. The small spot size maintained in the plasma channel allows $a_{0}$ to grow through temporal compression of the pulse and enables the threshold value for self-injection $\left(a_{0} \approx 3-4[8,15-17]\right)$ to be reached at $z \approx 25 \mathrm{~mm}$. Figure 5 shows that $a_{0}$ never exceeds 2 , and hence the conditions for self-injection are not reached, if the same laser pulses are input to a gas cell with a density corresponding to $P=80 \mathrm{mbar}$. The simulations show that with the plasma channel the peak laser power remains below $P_{c}^{\text {diff }}$ up to the point of self-injection. It is clear from these simulations that an external waveguide is necessary to achieve electron injection at these low densities and laser powers.

It is interesting to note that for $P=80$ mbar the length of the plasma channel after the point of self-injection is approximately equal to the dephasing length $L_{d} \approx 8 \mathrm{~mm}$, and is less than the pump depletion length $L_{p d} \approx 20 \mathrm{~mm}$, which would assist the generation of monoenergetic electron beams. Additional simulations with WAKE show that at higher (lower) initial pressures the conditions for electron injection are reached earlier (later), which can be expected to lead to broader electron energy spectra-as observed in Fig. 3-owing to acceleration over distances longer (shorter) than $L_{d}$.

Figure 4 shows that changes in the transverse profile of the input laser beam can have a significant effect on the total laser pulse energy required for injection and acceleration. The effect of the aperture on the propagation of the driving laser pulse is modeled in Fig. 5, which shows that without the aperture $a_{0}$ does not exceed 2 for an on-target total pulse energy of $2.5 \mathrm{~J}$ and hence that injection would not occur, as found experimentally. Additional simulations of the propagation in a plasma channel of laser pulses with a Gaussian transverse profile show that the propagation beyond the first few $\mathrm{mm}$ is relatively insensitive to the input beam spot size. We conclude that the reduced threshold observed with the apertured beam did not arise from better matching of the input spot size to the channel, but from an increase in the proportion of energy contained in low-order transverse modes.

In conclusion we have shown that plasma channels with matched spot sizes $w_{m}>w_{s f}$ can suppress diffraction sufficiently for a laser pulse with $P_{0}<P_{c}^{\text {diff }}$ to evolve to one which propagates stably with a spot size close to $w_{s f}$, and that this enables electron injection at lower laser powers and plasma densities than possible without a waveguide. We demonstrated this approach using plasma channels with $n_{e}(0)=1.8 \times 10^{18} \mathrm{~cm}^{-3}$ and $w_{m} \approx 35 \mu \mathrm{m}$ to generate quasimonoenergetic electron beams with energies greater than $500 \mathrm{MeV}$ with an input laser energy of only $2.5 \mathrm{~J}$, corresponding to a peak power of $30 \mathrm{TW} \approx 0.2 P_{c}^{\text {diff }}$ and $w_{s f}=12 \mu \mathrm{m} \approx 0.3 w_{m}$. It is interesting to note that, in experiments with the same laser and focusing optic (no aperture), but employing a gas-jet target, $12 \mathrm{~J}, 55 \mathrm{fs}$, 220 TW laser pulses were used to generate electron beams with energies up to $800 \mathrm{MeV}$ at an electron density of $5.7 \times$ $10^{18} \mathrm{~cm}^{-3}$ [7]. Hence, use of a plasma channel enabled the generation of electron beams with energies of $65 \%$ of those obtained with a gas jet, but using laser pulses of only $4 \mathrm{~J}$ (no aperture) or $2.5 \mathrm{~J}$ (with aperture). We also demonstrated that optimizing the quality of the input laser beam can significantly reduce the laser energy required. In Ref. [3] laser pulses of lower energy, but with better focal spot quality and shorter pulse duration, were guided in a plasma channel to generate electron beams with energies up to $1 \mathrm{GeV}$. The results of the present paper and those of [3] show that optimization of the plasma channel and the driving laser pulses are likely to lead to further improvements in the performance of the plasma accelerator.

\section{ACKNOWLEDGMENTS}

We acknowledge the support of the U.K. Engineering and Physical Sciences Research Council (Grant No. EP/ C005449), and the European Community-New and Emerging Science and Technology Activity under the FP6 "Structuring the European Research Area" program (project EuroLEAP, Contract No. 028514).

[1] T. Tajima and J. M. Dawson, Phys. Rev. Lett. 43, 267 (1979).

[2] C. G. R. Geddes et al., Nature (London) 431, 538 (2004).

[3] W. P. Leemans et al., Nature Phys. 2, 696 (2006).

[4] K. Nakamura et al., Phys. Plasmas 14, 056708 (2007).

[5] S. P. D. Mangles et al., Nature (London) 431, 535 (2004).

[6] J. Faure et al., Nature (London) 431, 541 (2004).

[7] S. Kneip et al., Phys. Rev. Lett. 103, 035002 (2009).

[8] W. Lu et al., Phys. Rev. ST Accel. Beams 10, 061301 (2007).

[9] S. Gordienko and A. Pukhov, Phys. Plasmas 12, 043109 (2005).

[10] D. J. Spence and S. M. Hooker, Phys. Rev. E 63, 015401 (R) (2000).

[11] A. Butler, D. J. Spence, and S. M. Hooker, Phys. Rev. Lett. 89, 185003 (2002).

[12] A. J. Gonsalves et al., Phys. Rev. Lett. 98, 025002 (2007).

[13] T. P. Rowlands-Rees et al., Phys. Rev. Lett. 100, 105005 (2008).

[14] Patrick Mora and Thomas M. Antonsen, Jr., Phys. Plasmas 4, 217 (1997).

[15] F. S. Tsung et al., Phys. Rev. Lett. 93, 185002 (2004).

[16] S.P. D. Mangles et al., Phys. Plasmas 14, 056702 (2007).

[17] S. P. D. Mangles et al., IEEE Trans. Plasma Sci. 36, 1715 (2008). 